\title{
An Infrared Small Target Detection Framework Based on Local Contrast Method
}

\author{
Zheng Cui, Jingli Yang*, Junbao Li, Shouda Jiang \\ Department of Automatic Testing and Control \\ Harbin Institute of Technology, Harbin 150080, China
}

\begin{abstract}
Small-target detection in infrared imagery used in forward looking infrared is an important task in remote sensing fields. It is important to improve the detection capabilities such as detection rate, false alarm rate,speed, ect. In this letter, a novel approach inspired by Human Visual System (HVS) is presented. First, block compressed sampling theory was used to compress the image and obtain the modulation map. Then, small abnormal regions in the modulation map were detected by high speed local contrast method and defined as candidate targets. Experimental results show the proposed algorithm pursue good performance in detection rate, false alarm rate and speed simultaneously.

Keywords: small target detection, modulation map, human visual system, high speed local contrast method, machine learning
\end{abstract}

\section{Introduction}

Infrared(IR) small-target detection plays a critical role in large amounts of practical projects such as infrared warning and defense alertness, especially in forward looking infrared (FLIR) equips the unmanned aerial vehicle (UAV) [? ]. FLIR has the features of small volume, light weight, low power, high stability and so on. These characteristics led to their processing equipment weak in calculation ability. Meanwhile, the processing equipment may need a faster processing speed(generally requires processor processes 25-80 frames per second) because of the battlefield situation is changeable. In conclusion, algorithm used in this situation requires not only the speed but also accuracy.

Various algorithms have been developed in the past few decades[? ][? ][? ]. Conventional small target detection methods such as top-hat filter [? ], max-mean/max-median filter[? ], highpass filters based on LS-SVM[? ], nonparametric regression method[? ] and two-dimension minimum mean square error(TDLMS) method[? ] are widely used to reduce the background clutters. However, median filter is ineffective when the image is heavily cluttered; high-pass filter works well only for point target detection in images with homogeneous background; the result of top-hat filter is bad, when the target shape is unknown. Using of the brightness contrast of target and its neighborhood, Chen et al[?

\footnotetext{
${ }^{*}$ Corresponding author.

Email address: jinglidg@hit.edu.cn (Jingli Yang) 
]. propose target detection methods based on local contrast measure (LCM). But the speed of the method is unbearable. In recent years, a series of simple and fast algorithm based on Fourier Transform was proposed, such as spectral residual(SR)[? ], phase spectrum of quaternion fourier transform(PFT)[? ] ,hypercomplex fourier transform(HFT)[? ]. Significant in the field of target detection based on frequency domain method is very different with other methods. It transforms the airspace information to the frequency domain, and defines significant target and testing in the frequency domain. But it has been proven that the essence of SR and PFT is equal to the high-pass filter in frequency domain. HFT is more suitable for the big target.

So these methods may fail in certain circumstances, e.g., ground-sky background [? ]. This kind of situation is common in the UAV view which has a high demand on detection speed. The targets in this situation have a recognizable shape[? ] but a size of small target and easily overlapped by vegetation, roads, rivers, bridges. In view of the above reasons traditional algorithm produce huge false alarm rate in result and can't satisfy the detection speed. In order to design an appropriate method, a small target detection method inspired by the human visual system(HVS) has been designed in this paper. HVS is a kind of layered image processing system consisting of optical system, retina and visual pathways, which is nonuniform and nonlinear. In this paper, we hope to propose a multilayer framework. Our framework may drive one layer by its upper layer to improve its speed and ensure the accuracy of the algorithm by local-regional complex intelligent computing. The rest of this paper is organized as follows. In section 2, we describe the framework of the proposed algorithm and give a brief analysis. In section 3, we present the experimental results. Section 4 is the conclusion of this article.

\section{Layered Algorithm Based on LCM}

\subsection{Layered Framework}

Here is the proposed framework inspired by HVS with three layers (See Fig1). Human visual system(HVS) divides the scene into small patches and select important information through visual attention selection mechanism to make it easier to understand and analyze. On the other hand, as a component of low-level artificial vision processing, it facilitates subsequent processing by reducing computational cost, which is a key consideration in real-time applications.

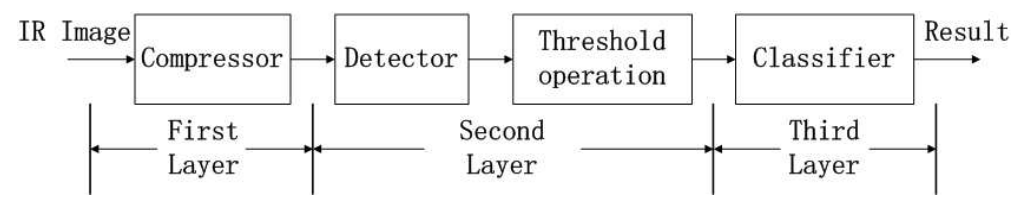

Figure 1: The proposed framework inspired by human visual system.

Due to the data collected by UAV need to be transmitted to the centre of command, data compression is required. Inspired by HVS, we used block compressed sampling[? ] theory to compress the image and found that the informa- 
tion of block has not been fully utilized. Therefore, in the first layer, the framework processes information compressed and obtain modulation map. Details will be described in section 2.2.

In the second layer, We use a traditional target detection algorithm when working with the modulation map. Small infrared target has no texture information or direction information but a higher brightness comparing with the background. That means contrast is the most important quantity encoded in the streams of visual system. With these considerations in mind, we select the contrast of brightness as a standard to decide whether pay attention to the areas or not. In reference[? ], Chen et al. propose target detection methods based on local contrast measure (LCM) .Because the speed of the traditional local contrast method is unbearable and the method do not apply to abnormal morphology targets, we proposed high speed local contrast method(HSLCM) to improve it. Details will be described in section 2.3. Candidate targets can be got through HSLCM followed by threshold operation.

After that, some complex processing is performed to extract targets from candidate targets in the third layer.

In view of background clutters are similar to the infrared targets in the size and shape, it is very difficult for traditional algorithms to distinguish them[? ]. The machine learning method, making use of the statistical features of targets, will be very effective for this kind of classification problem. In this paper, we use support vector machines (SVM)[? ] classifier to finish the work. Other machine learning methods could be also work here. However, since the original target templates and background templates are manually selected from the first frame, the training samples will be few in number. SVM is advantageous to deal with problems with high dimensions, small samples and uncertainty. Details will be described in section 2.4.

We believe that these three stages would get a better balance algorithm[? ].

\subsection{First layer: obtain modulation map}

In the first layer,the framework divides the scene into small patches and uses block compressed sampling theory to compress the image. The block compressed sampling theory independently does measurement and reconstruction using the same measurement matrix for each image block of the same size.

Consider an image with $r \times l$ pixels in total, and we want to take n CS measurements. The image is divided into small blocks with $r_{b} \times l_{b}$ size, thus each row has $l / l_{b}$ blocks and each line has $r / r_{b}$ blocks. The size of the block is close to the size of the target ( 0.15 of the total size of the image). A window is slid on the IR image from up-to-down and left-to-right at a specific step, and a series of sub-blocks is obtained (seq 1, image I is divided into $\left.B_{1}, B_{2}, \ldots B_{n}, n=\frac{r \times l}{r_{b} \times l_{b}}\right)$ and compressed with the same operator.

$$
I=\left[\begin{array}{cccc}
g_{11} & g_{12} & \cdots & g_{12} \\
g_{21} & \ddots & & \vdots \\
\vdots & & \ddots & g_{(r-1) l} \\
g_{r 1} & \cdots & g_{r(1-1)} & g_{r l}
\end{array}\right]=\left[\begin{array}{ccc}
B_{1} & \cdots & B_{l / l_{b}} \\
\vdots & \ddots & \vdots \\
B_{n-l / l_{b}+1} & \cdots & B_{n}
\end{array}\right]
$$


where $g_{i j}$ represents the gray value of the image I location on the ith row and the jth line $(i=1,2 \ldots r, j=1,2 \ldots l)$. Let $x_{p}$ represent the vectorized signal of the $\mathrm{pth}(p=1,2 \ldots n)$ block. The corresponding output random project vector, $y_{p}$, can be written as

$$
y_{p}=\Phi_{B} \times x_{p}
$$

The entire image can be written as

$$
y=\left[\begin{array}{c}
y_{1} \\
\vdots \\
y_{n}
\end{array}\right]=\left[\begin{array}{lll}
\Phi_{B} & & \\
& \ddots & \\
& & \Phi_{B}
\end{array}\right]\left[\begin{array}{c}
x_{1} \\
\vdots \\
x_{n}
\end{array}\right]
$$

where $\Phi_{B}$ is a $m \times\left(r_{b} \times l_{b}\right)$ matrix, with $m \ll\left(r_{b} \times l_{b}\right)$. In our current work, $\Phi_{B}$ is an orthonormalized i.i.d Gaussian matrix[?].

We use the compressed data, $\mathrm{y}$, to construct a modulation map. The modulation map has a $r / r_{b} \times l / l_{b}$ dimension, which is the same quantity of the sub-blocks. As an example, we put all output random project vector's first values on the corresponding position to constitute the first modulation map (seq4; the modulation map is comprised of $\left.y_{1}^{1}, y_{2}^{1}, \ldots y_{n}^{1}, n=\frac{r \times l}{r_{b} \times l_{b}}\right)$.

$$
\operatorname{map}=\left[\begin{array}{ccc}
y_{1}^{1} & \cdots & y_{l / l_{b}}^{1} \\
\vdots & \ddots & \vdots \\
y_{n-l / l_{b}+1}^{1} & \cdots & y_{n}^{1}
\end{array}\right]
$$

\subsection{Second Layer Processing Method}

A real IR small target usually occupies a small area, thus a sub-block may contain all or most small targets or the complete background, which can be denoted as the target block (TB) and background block (BB), respectively. Because real IR small targets are usually brighter than background, the modulation values calculation by TB are usually higher than the modulation values calculation by BB, thus there is a contrast between the two kinds of values and traditional algorithms may remain valid in modulation maps. We therefore used a HSLCM algorithm when working with the modulation map. After that, a threshold operation was used to select the suspected area containing the target zone.

The details of HSLCM will be introduced in this section. Here are some basic definitions.

As shown in Fig.2, u denotes the local region, and the area between $\mathrm{u}$ and $\mathrm{v}$ is the local background region. Moreover, the modulation map is denoted by $\mathrm{w}$. In this case, the windows v can move on the w. 
Based on the aforementioned preparation, we can find that different image patches of size $\mathrm{v}$ can be obtained by moving the window $\mathrm{v}$ on the modulation map. Furthermore, u can move on v. Consequently, the obtained image patches can be given by nine cells (see Fig.3).

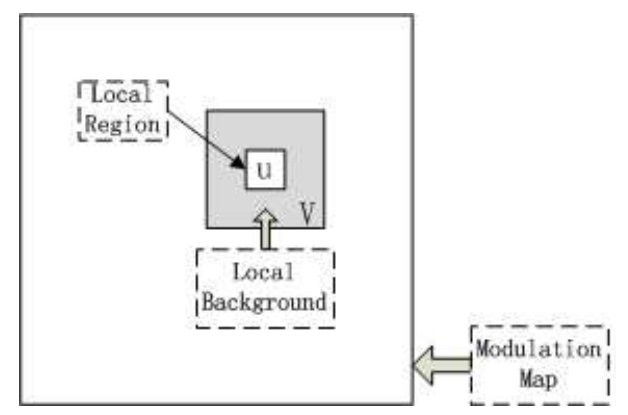

Figure 2: Multiple windows.

\begin{tabular}{|l|l|l|}
\hline 1 & 2 & 3 \\
\hline 4 & 5 & 6 \\
\hline 7 & 8 & 9 \\
\hline
\end{tabular}

Figure 3: Image patch obtained by sliding window.

Through the analysis of LCM algorithm, we find out that several average operations and calculated pixel by pixel are both time consuming. The saliency c of LCM is defined as follows.

$$
c_{L C M}=\frac{g_{\max }^{2}}{m_{\max }}
$$

$g_{\max }$ expresses the maximum of the gray value of the central cell and $m_{\max }$ express the maximum gray mean of the cells in the local background. In order to increase computation speed of LCM, we try to simplify the model. Like LCM, HSLCM describes a pixel (position) by generating a signal value.

Instead of the maximum gray mean, we use the average of all neighboring cells gray values to be the comparison, which makes the saliency $\mathrm{c}$ as follows.

$$
c_{H S L C M}=\frac{g_{\max }^{2}}{m_{\text {all }}}
$$

$m_{\text {all }}$ can be defined as follows.

$$
m_{\text {all }}=\frac{1}{N} \sum_{i=1, i \neq 5}^{9} \sum_{j=1}^{N} g_{i j}
$$


where $\mathrm{N}$ is the number of the pixels in the $i_{t h}$ cell and $g_{i j}$ is the gray level of the $j_{t h}$ pixel in the $i_{t h}$ cell. Cell 5 is the central cell, so $\mathrm{i}$ is not equal to 5 .

Obviously the new method could be much faster than the original method. Then we analyze amount of calculation of the two algorithms.

Assuming the size of the cell to a $\mathrm{x}$ a and image pixels to $\mathrm{N}$. Each pixel has a * a 8 times of add operation, 8 times of division operation and a $* a+7$ times of comparison operations needs to be done for traditional LCM algorithm. But for HSLCM algorithm, only a * a * 8 times of add operation, 1 time of division operation and a $*$ a times of comparison operations needs to be done. Due to the 7 times of division operation and 7 times of comparison operations reduced for each pixel, the new method could be much faster than the original method. Therefore, the amount of calculation for a picture will reduced by $7 * \mathrm{~N}$ times of division operation and $7 * \mathrm{~N}$ times of comparison operations.

HSLCM algorithm also brings some influence to calculation accuracy which will be analyzed from the configuration of the target. Due to the optics point spread function (PSF) of the thermal imaging system at a long distance, there are often two kinds of small target shape, circular and elliptic. For the convenience of analysis, we do some assumptions as the prerequisite. First of all, the small target is assumed to be a 25 pixels (generally considered small target accounts for less than $0.15 \%$ of the total size of the image, our images have $128 \times 128$ pixels)spot. In the ideal case, the size of cell should be the same as the target size.

Average of all gray values in 8 neighboring cells is considered approximate to be background brightness. Refer to the commonly used definition, the contrast of target and background is as follows:

$$
\eta=\frac{G_{t}-G_{b}}{G_{b}}
$$

Where $G_{t}$ is the grayscale average of targets, and $G_{b}$ is the grayscale average of background. So the average of all

8 neighboring cells gray values can be consider as $G_{b}$ and evaluate as $\frac{G_{t}}{1+\eta}$. The contrast ratio of dim small targets and the background is generally considered as 0.15 or less than it.

Then,we discussed three kinds of circumstances which difference in locations of center pixel relative to a target.

1, The central part is almost all targets(central part of a circular target).

2, Part of the center cell is targets(edge of a circular target).

3 , There is no target in the center cell(outside of a circular target).

Three of the schematic diagram is as follows(see Fig.4).

Using the new method has no effect on (a).

For circumstance (b), the significant degree of cell 5 is strengthened. Apparently, cell 4 is the brightest in all surrounding cells. So the significant degree of original LCM algorithm is as follows:

$$
c_{L C M} \approx \frac{G_{t}^{2}}{\frac{G_{t}+G_{b}}{2}}=\frac{(1+\eta)^{2} G_{b}^{2}}{\left(1+\frac{\eta}{2}\right) G_{b}}=\frac{2(1+\eta)^{2}}{2+\eta} G_{b}
$$




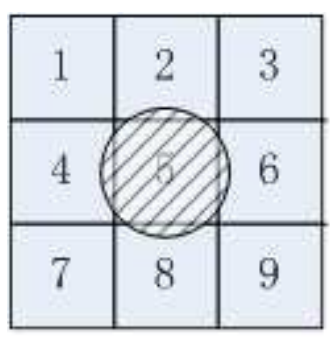

(a)

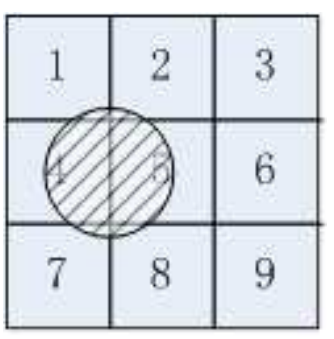

(b)

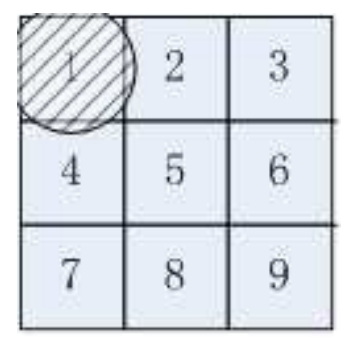

(c)

Figure 4: different parts of a circular target. (a)central part of a circular target. (b)edge of a circular target. (c)outside of a circular target.

Based on our assumptions before, the significant degree of HSLCM algorithm is as follows.

$$
c_{H S L C M} \approx \frac{G_{t}^{2}}{G_{b}}=(1+\eta)^{2} G_{b}
$$

$c_{H S L C M} \geq c_{L C M}$ help us to come to a conclusion that the effect of $c_{H S L C M}$ is better than $c_{L C M}$.

In the case of (c), the original method has an inhibiting effect to the significant degree of cell 5 , but improved method has not. Here follows our analysis. Obviously, the brighter the around, the stronger the inhibition. Continue to use previous assumptions, the significant degree of original LCM algorithm is as follows.

$$
c_{L C M} \approx \frac{G_{b}^{2}}{G_{t}}=\frac{G_{b}^{2}}{(1+\eta) G_{b}}=\frac{G_{b}}{1+\eta}
$$

The significant degree of HSLCM algorithm is as follows.

$$
c_{H S L C M} \approx \frac{G_{b}^{2}}{G_{b}}=G_{b}
$$

$c_{L C M} \geq c_{H S L C M}$ help us to come to a conclusion that the effect of $c_{L C M}$ is better than $c_{H S L C M}$.

Based on the analysis above, we can come to the conclusion that HSLCM has a higher marginal significant degree and also a higher significant degree of the background outside of the target. A higher marginal significant degree means the target is more unambiguous, but A higher significant degree of background means oppositely. We can compare the ratio between the two significant degree to do some simple quantitative analysis. Ratios of the two algorithms are as follows.

$$
\begin{gathered}
m_{L C M}=\frac{\frac{2(1+\eta)^{2}}{2+\eta} G_{b}}{\frac{G_{b}}{1+\eta}}=\frac{2(1+\eta)^{3}}{2+\eta} \\
m_{H S L C M}=\frac{(1+\eta)^{2} G_{b}}{G_{b}}=(1+\eta)^{2}
\end{gathered}
$$

$m_{L C M} \geq m_{H S L C M}$ means targets of $c_{L C M}$ are more obvious than $c_{H S L C M}$. Thus, HSLCM may be more sensitive to threshold in the following operation. 
After analyzing of the above three conditions, we discuss targets with noncircular shape. The small target is assumed to be a spot with a shape of the ellipse. Be similar with the circular target, we discussed four kinds of circumstances (See Fig.5).

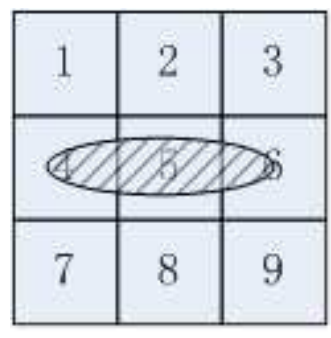

(a)

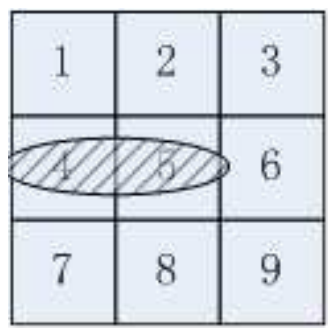

(b)

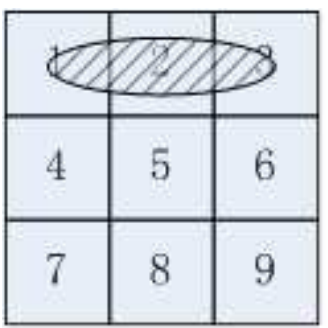

(c)

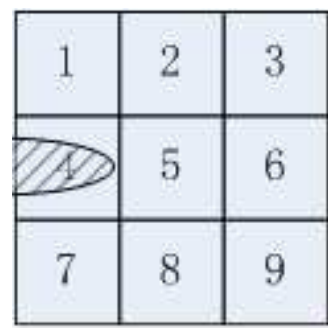

(d)

Figure 5: different parts of a ellipse target. (a)central part of a ellipse target. (b)edge of a ellipse target. (c)outside of a ellipse target's long side.(c)outside of a ellipse target's short side.

Due to the influence of the shape, in situation (a), elliptic targets might have more parts on surrounding cells. Therefore, reference formula 5, the denominator of $c_{L C M}$ will be affected by the part of the target locate in cell 4 or cell 6.However, reference formula 6 , background brightness would has a more important impact on the denominator of $c_{H S L C M}$. It causes the denominator of $c_{H S L C M}$ smaller than the denominator of $c_{L C M}$ which means $c_{H S L C M} \geq c_{L C M}$.

For circumstance (b), we can get a similar conclusion that the significant degree of LCM algorithm is obviously smaller than the significant degree of HSLCM algorithm. It makes HSLCM easier to enhance the target pixel brightness.

In both case (c) and (d), the denominator of $c_{L C M}$ will be affected by the part of the target. However, the denominator of $c_{H S L C M}$ is almost the same level as background brightness. That means the significant degree of LCM algorithm is smaller than the significant degree of HSLCM algorithm.

The conclusion of ellipse target is similar with the circular target's. however circumstance (b) of ellipse target has a bigger size. That means HSLCM is more suitable for small target with a shape of the ellipse. For target in forward looking infrared image as mentioned in introduction, HSLCM has a good adaptability to get the significant map.

After the final significant map be obtained, a threshold operation is used to pick out the most salient points. Next, define the thresholds as followed.

$$
T=\frac{1}{N_{I}} \sum_{j=1}^{N_{I}} S_{j}+k *\left(\operatorname{Max}\left(S_{j}\right)-\frac{1}{N_{I}} \sum_{j=1}^{N_{I}} S_{j}\right)
$$

Where $S_{j}$ represents the saliency value of the $j_{t h}$ pixel,$N_{I}$ is the number of the pixels of the image. $\mathrm{k}$ is a parameter from 0 to 1 and be used to control the threshold changing from $\frac{1}{N_{I}} \sum_{j=1}^{N_{I}} S_{j}$ to $\operatorname{Max}\left(S_{j}\right)$. We only deal with those areas above the threshold. Setting threshold can effectively reduce the number of salient areas, thus reduce the amount of calculation. 


\subsection{Third Layer: support vector machines classifier}

The thought of detection algorithm based on machine learning is converting a target detection problem into a pattern classification problem. The core of this detection algorithm is making full use of prior knowledge.

As a preparation for classified correctly, it is important to obtain training sets by using the statistical features of the small targets. An accurate sample library of the target and the background is the key of the correct classification. At initialization, the first target template is manually selected from the first frame. We pick up real targets and locate target's centers. The first target template will be defined as a vector which is made from a center pixel with its $(16 \times 16)$ neighbors. The remaining target templates are created by perturbating a few pixels(in our paper the offset is from one pixel to four pixels) in four directions(up,down,left,right) at the corner points of the first template in the first frame. Then we can get 100 centres(including the first one with no offset) and 100 samples. Background templates are manually selected from the salient regions of the first frame. We also selected 100 samples.

Considering the target shape may have a slight change in the process of moving, which because of the change of direction and attitude, we could update the training set to make the classifier more intelligent. This process includes join target and false target of the testing image into the training set respectively.

The penalty factor of SVM classifier is 30. SVM classifier using the gaussian kernel and its parameter is 0.01 . All parameters are selected according to the experience.

Due to the SVM classifier can separate target from clutters, the accuracy can be improved.

\section{EXPERIMENTAL RESULTS AND ANALYSIS}

Experiments on the real IR image sequence have been done. The experimental data including several group of images with a resolution of $(128 \times 128)$, obtained from United States Army Aviation and Missile Command (AMCOM). AMCOM was formed on a provisional basis on 17 July 1997 by merging the U.S. Army Missile Command(MICOM) and the U.S. Army Aviation and Troop Command(ATCOM). By Permanent Orders 344-1, dated 9 December 1996, the U.S. Army Materiel Command (AMC) directed that AMCOM be established at Redstone Arsenal on a permanent basis effective 1 October 1997. MICOM and ATCOM had previously exercised their respective responsibilities in missilery and aviation in joint efforts to develop certain airborne missile systems to support the soldier in the field. Due to the ground background is more complex than the sky background, the test success rate of one type of missile to the target set in the sky is much higher than target set in the ground. AMCOM provides 51 actual FLIR image sequence, including more than 16000 frames, for the researchers to study of small target detection and tracking algorithm of ground background. All experiments were implemented by MATLAB software on a PC with 4-GB memory and 3.2-GHz Intel i5 dual processor. Here follows our experiment design. The experiment design involved two stages. First is the contrast between HSLCM and LCM. We may verify the correctness of our views in section 2.3. Second is the result of the performance of our framework. 


\subsection{The performance of HSLCM}

In section 2.3, small targets with circular and elliptic shapes have been analyzed respectively. Thus, we choose two groups of images(see Fig.6) separately verify these two analysis result. The real targets are marked in two examples. Target in Fig.6(a) is a Civilian truck. Image shows the front view of the truck. Targets in Fig.6(b) are two different vehicles. There is a tank on the left and an armored vehicle on the right. Image shows the side view of the tank and armored vehicle.

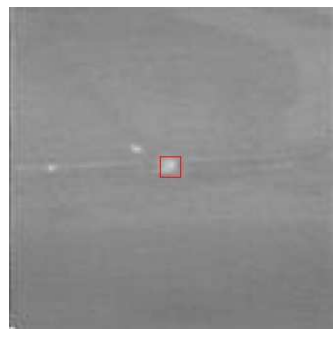

(a)

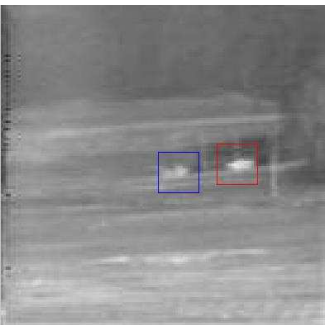

(b)

Figure 6: (a)circular target. (b)elliptic target.

First, we verify the conclusion about circular target. The same image in Fig.6(a) is used to be the example. Here follows the figures of simulation results(see Fig.7 and Fig.8).In these figures, (a) is the saliency map. (b) is the result after the same threshold operation. (c) is the detection result and all the targets are labeled by asterisks. The cell size is set to $(5 \times 5)$. Parameter $\mathrm{k}$ in threshold is set to 0.25 .

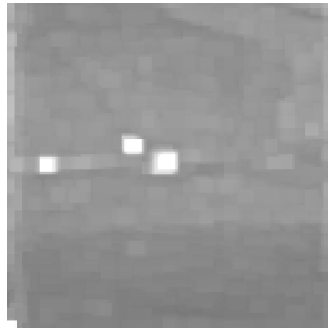

(a)

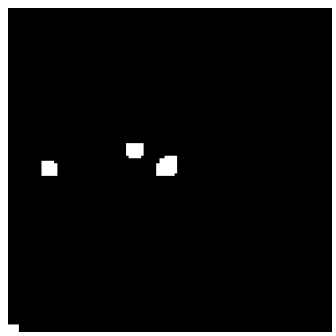

(b)

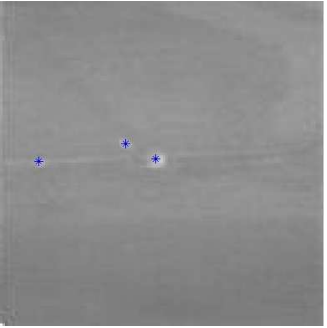

(c)

Figure 7: high speed local contrast method calculation. (a)saliency map. (b)result of threshold operation. (c)detection result.

It can be seen that the effect of HSLCM is very approximate to LCM.

To reveal the advantages of the proposed algorithm to the other three algorithms, set algorithms threshold to different values and compare the detection rate [true positive rate (TPR) defined in (16)][? ] in Fig.9. Experimental results show that the parameter $\mathrm{k}$ in (12) is suitable to be set from 0.1 to 0.3 in our work. We can get high TRP with all kinds of algorithms by selecting this parameters section so as to meet the requirements. 


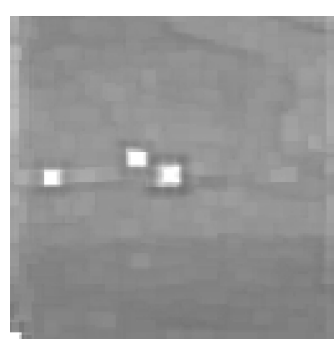

(a)

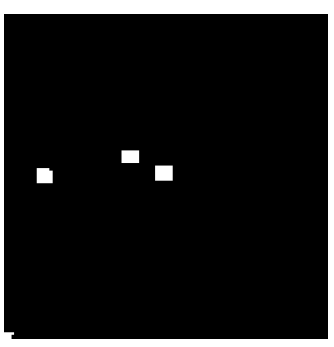

(b)

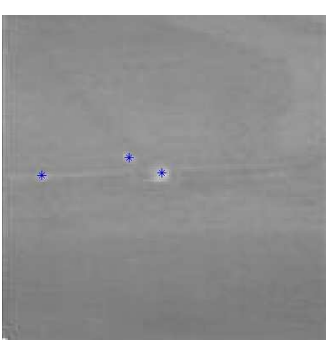

(c)

Figure 8: local contrast method calculation. (a)saliency map. (b)result of threshold operation. (c)detection result.

TPR defined as

$$
T P R=\frac{\text { Quantity of true } T B \text { detected in images }}{\text { Quantity of true TB existing in images }} * 100 \%
$$

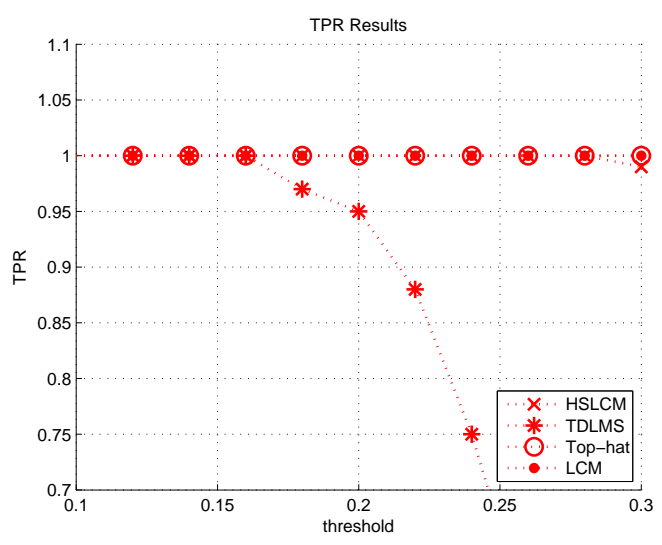

Figure 9: true positive rate of circular target using four methods.

From the experimental results, we can get the conclusion that HSLCM has almost the same performance with LCM. Just like our analysis, the TPR of HSLCM achieve 99\%. Therefore, for circular target, HSLCM palys a good effect. Our analysis has been affirmed.

Then, we verify the conclusion about elliptic target. The same image in Fig.6(b) is used to be the example. Simulation results are shown in Fig.10 and Fig.11.In these figures,we use a same cell size and parameter k.

We do the same operation as circular target. Experimental results show that the parameter k in (12) is suitable to be set from 0.1 to 0.3 in our work(See Fig.12).

From the experimental results, we can get the conclusion that HSLCM and LCM can achieve the best performance in TPR.

Although the targets are successfully detected, there are many false alarms existed. We define false alarm rate [false positive rate (FPR) defined in (17)] to measure it. 


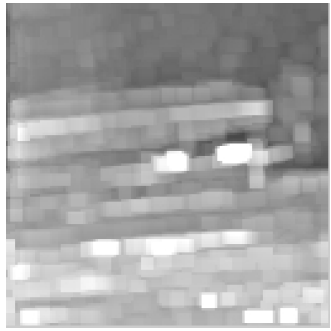

(a)

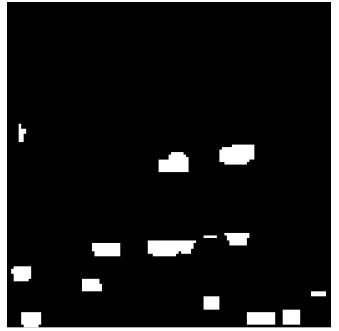

(b)

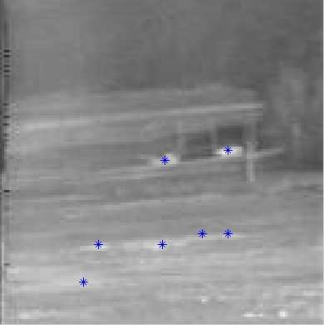

(c)

Figure 10: high speed local contrast method calculation. (a)saliency map. (b)result of threshold operation. (c)detection result.

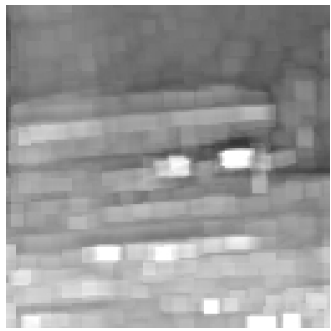

(a)

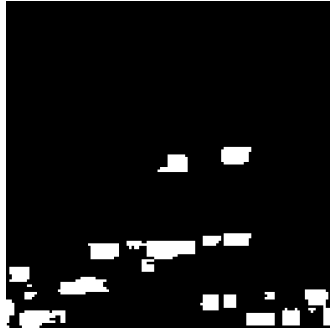

(b)

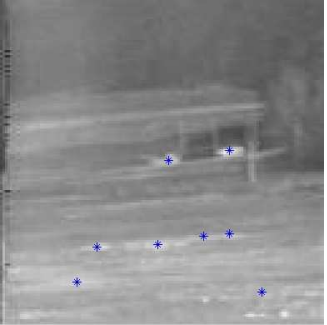

(c)

Figure 11: local contrast method calculation. (a)saliency map. (b)result of threshold operation. (c)detection result.

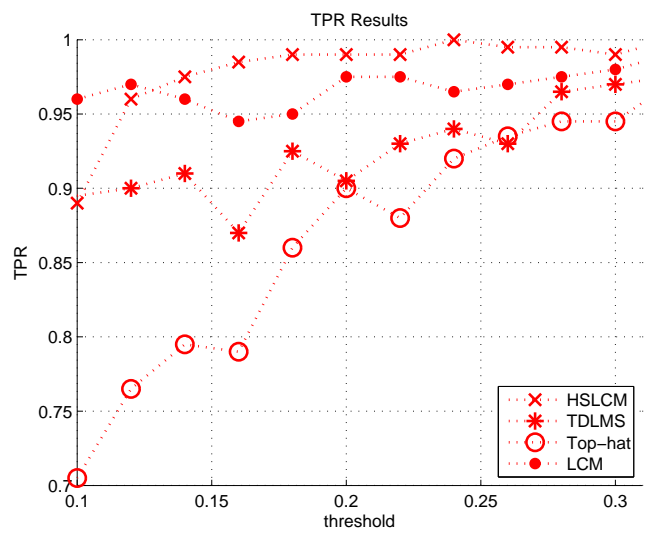

Figure 12: true positive rate of elliptic target using four methods. 
FPR defined as

$$
F P R=\frac{\text { Quantity of false TB detected in images }}{\text { Quantity of true TB existing in images }} * 100 \%
$$

Here follows the FPR of circular target and elliptic target using four methods.

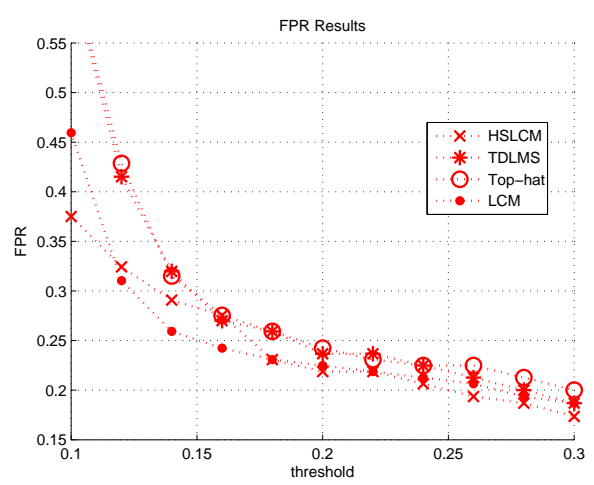

(a)

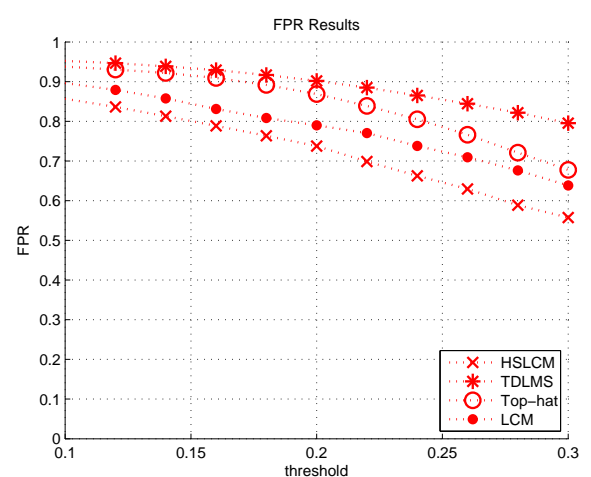

(b)

Figure 13: comparison of false positive rate. (a)false positive rate of circular target. (b)false positive rate of elliptic target.

From the experimental results, we can get the conclusion that HSLCM has the lowest FPR in all four methods.

Then, we compare the average computational cost of these detection methods for 100 images (see Fig.14). From the experimental results, we can get the conclusion that top-hat method has the fastest computing speed in these four methods. HSLCM algorithm is just a few seconds faster than TDLMS algorithm. The method of sliding window of LCM method based on pixel mobile makes its calculation speed slowest.

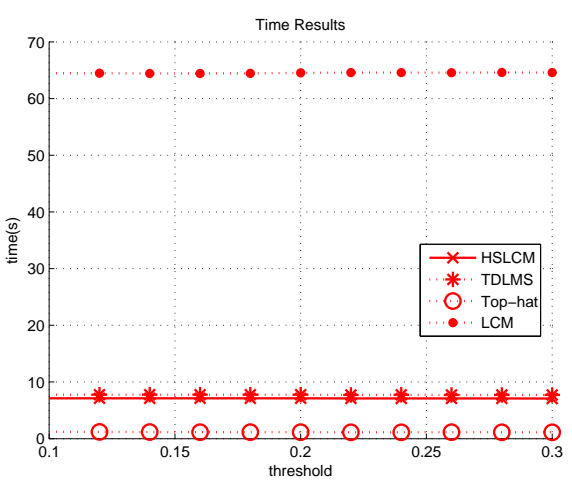

(a)

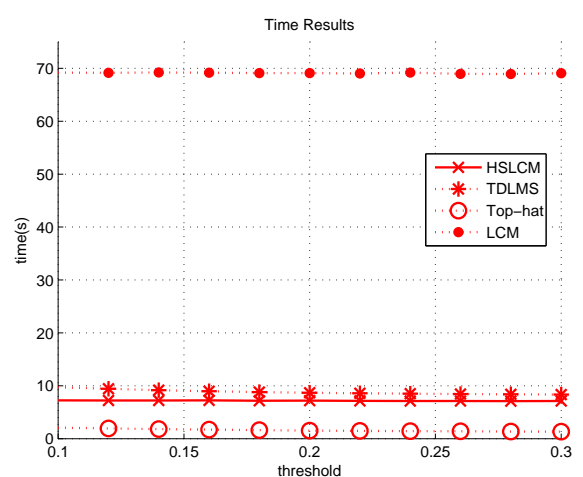

(b)

Figure 14: comparison of several methods' time.(a)time for circular target. (b)time for elliptic target. 


\subsection{The performance of our framework}

Although the targets are successfully detected, the false alarms and the slow detection speed are both unacceptable. We hope to reduce the false alarm rate and detection time by using the our framework in section 2.1.

Experimental results using the proposed framework are shown in Fig.15 and Fig.16.

Fig.15(a) and Fig.16(a) is the detection result of HSLCM. Fig.15(b) and Fig.16(b) is the detection result of the proposed framework. Obviously, SVM classifier can effectively distinguish the false target and the real goal.

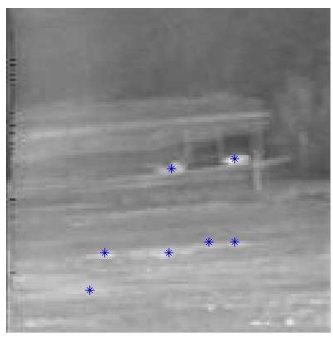

(a)

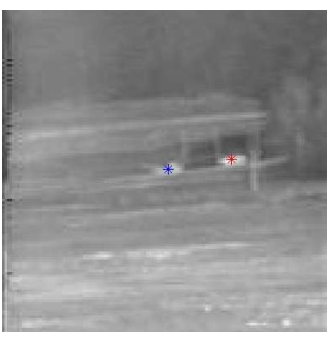

(b)

Figure 15: comparison between detect results of high speed local contrast method and our framework for elliptic target detection. (a)detect result of high speed local contrast method. (b)detect result of our method.

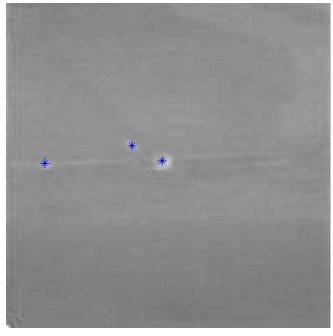

(a)

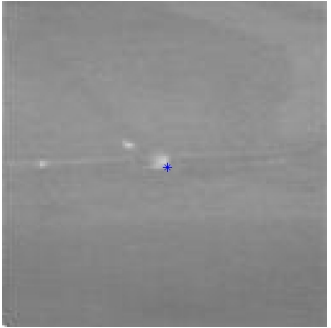

(b)

Figure 16: comparison between detect results of high speed local contrast method and our framework for circular target detection.(a)detect result of high speed local contrast method. (b)detect result of our method.

To be fair, we use different preprocessing algorithms to verify the proposed framework. We choose 0.3 as threshold to compare the performance of all methods and list performance index in table 1(circular target) and table 2(elliptic target). We mainly analyze the elliptic target. From the experimental results, we can get the conclusion that the proposed framework has a huge increase in detect efficiency and reduce the FPR. HSLCM algorithm has a same TPR as LCM, a low FPR and the fastest operation time.

It was found that modified threshold does not affect TPR and FPR but only affect the computation time. This is because the preprocessing steps of this method only does operation on modulation image. We found that only the size of cell in HSLCM played a key role. So we set method cell size to different values and compare the TPR in Fig.17(a), 


\begin{tabular}{lccc} 
Table 1: Result of four using in our framework for circular \\
\cline { 2 - 4 } Method & TPR & FPR & time \\
\hline HSLCM & 0.8187 & 0 & 1.843 \\
TOP-HAT & 0.9688 & 0.2381 & 2.388 \\
TDLMS & 0.625 & 0.3195 & 1.98 \\
LCM & 0.5813 & 0.0303 & 4.618 \\
\hline
\end{tabular}

Table 2: Result of four using in our framework for elliptic target.

\begin{tabular}{lccc}
\hline Method & TPR & FPR & time \\
\hline HSLCM & 0.99 & 0.1304 & 2.637 \\
TOP-HAT & 0.98 & 0.115 & 3.138 \\
TDLMS & 0.93 & 0.5169 & 2.74 \\
LCM & 0.99 & 0.1525 & 6.334 \\
\hline
\end{tabular}

FPR in Fig.17(b). Experimental results show that the cell size is suitable to be set from 1 to 3 in our work. We can get high TRP and low FPR at the same time.

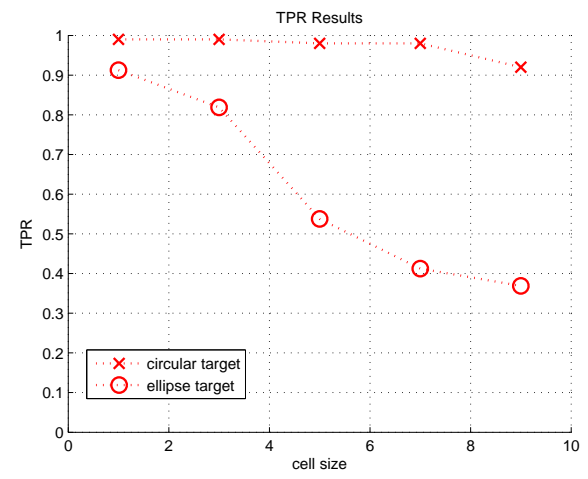

(a)

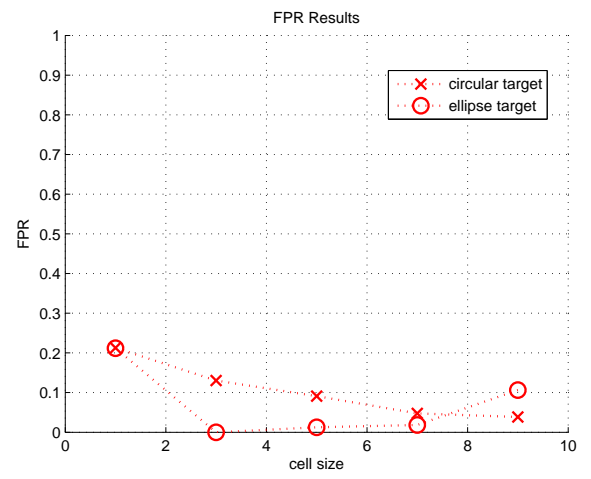

(b)

Figure 17: different cell size.(a)TPR change with cell size. (b)FPR change with cell size.

\section{CONCLUSION}

In this paper, an effective small target detection framework based on LCM is presented. Motivated by HVS, the key idea of the proposed method is to use the HSLCM to preproccess a modulation image obtained by block compressed 
sensing algorithm and use a threshold operation to choose the most significant areas. Then SVM classifier separates targets from background clutters. Experimental results show that the proposed algorithm is robust to resist pseudo targets and can achieve a high detection rate in less than $0.02 \mathrm{~s}$ with a fast calculation speed. It is worth noting that the FPR of proposed algorithm is far below other algorithms. This algorithm can be either directly used in single-frame target detection or used as a foundation module in sequential target tracking for real-time applications.

Based on the above conclusions, the proposed algorithm has a balanced performance on TPR, FPR and detection time.

\section{Acknowledgement}

This work is supported by the Fundamental Research Funds for the Central Universities(Grant No.HIT.NSRIF.2017014) and Program for New Century Excellent Talents in University under Grant No. NCET-13-0168 and National Science Foundation of China under Grant No.61371178.

\section{References}

